\title{
Sticking to an Old Definition of Ventilator-Associated Pneumonia Is Not Old-Fashioned
}

Intensive care medicine developed as a consequence of the poliomyelitis epidemic of the 20th century, when widespread mechanical ventilation was required for hundreds of victims. ${ }^{1}$ Since then, the technology available to ventilate the critically ill patient has become more sophisticated but also complex, and the importance of ventilation as a lifesaving tool in today's health care and ICU is without question. However, one fifth of the patients in the ICU, especially those ventilated invasively, acquire at least one hospital-acquired infection. Ventilator-associated pneumonia (VAP), occurring in mechanically ventilated patients following intubation, is certainly one of the most dramatic and prevalent types of nosocomial ICU infection. ${ }^{2}$ The incidence of VAP is high, in some studies occurring in even up to $27 \%$ of ventilated ICU subjects. ${ }^{2,3}$ Its occurrence is associated with increased length of ICU and hospital stay, hospital mortality, and financial burden..$^{2,3}$

In 2005, the American Thoracic Society defined VAP as a bacterial pneumonia present in patients receiving mechanical ventilation for at least $48 \mathrm{~h}$ combined with an infiltrate on the chest radiograph, signs of infection, and detection of a bacterial causative agent. ${ }^{4}$ However, diagnosis of VAP remains a very challenging issue. More particularly, the exact role of the microbiological detection in VAP is a matter of debate. In case of such a suspected pulmonary infection in ventilated patients, respiratory samples should be collected. However, since endotracheal tubes are colonized by bacteria within $6 \mathrm{~h}$ after intubation and ventilation initiation, many of these cultures should be considered false-positive, rather than reflecting rapid contamination/colonization without pathogenicity. ${ }^{5}$ Cultures may take days to become positive and have limited sensitivity, especially in patients already receiving antibiotic therapy. In one of 3 patients with VAP, bugs are detected by staining methods but cannot be cultured, also influenced by previous antibiotic therapy. ${ }^{2,3}$ Moreover, these staining techniques on

Dr Jorens has disclosed no conflicts of interest.

Correspondence: Philippe G Jorens MD, PhD, Department of Critical Care Medicine, Antwerp University Hospital and University of Antwerp, Wilrijkstraat 10,2650Edegem, Belgium.E-mail:Philippe.Jorens@uza.be.

DOI: $10.4187 /$ respcare.04736 biological material are unreliable to differentiate between bacterial subclasses. The impact of inadequate or delayed initiation of correct intravenous antibiotic treatment is huge: VAP mortality can increase from $29.2 \%$ to as high as $63.5 \%$, or such inappropriate care may

See the Original Study on Page 269

cause up to 16.4 additional days in hospital ${ }^{6,7}$ All of these findings provide the rationale for a quick identification of the bacteria in patients with clinical signs of VAP in an effort to improve the outcome of these patients. Specific and effective use of antibiotics, defined as coverage of all of the identified pathogens by the antimicrobial therapy administered at the time of VAP clinical diagnosis, are therefore highly desirable; this has been proven to limit ventilation duration, mortality, ecological disturbances (antibiotic resistance), and again economic costs ${ }^{2,3}$ As a consequence, VAP is frequently caused by bacteria that are highly resistant to the currently available antibiotics, further hampering treatment and thus increasing the already unacceptable morbidity, mortality, and costs.

The exact and fast identification of the patient with or at risk for VAP is therefore necessary but challenging. (1) The clinical signs of VAP lack specificity and sensitivity. (2) Current practice frequently necessitates an invasive technique to recover bacteria from deep tissue sites, such as lung lavage fluid, sputum, endotracheal aspirate, or blood. (3) All of the laboratory techniques used to identify the presence and type of pathogenic bacteria are hampered by an important time delay. Consideration should therefore be given to the development and validation of a good definition of VAP to distinguish between patients with or without VAP or even those at risk or not at risk for VAP.

Over the past few years, several groups have raised doubts about the validity of the original and traditional VAP definition. The Centers for Disease Control and Prevention (CDC) have recommended not taking the duration of ventilation (longer than $48 \mathrm{~h}$ ) into account. ${ }^{8}$ Because accurate surveillance of VAP is hampered by subjective diagnostic criteria, the CDC have also proposed a novel surveillance paradigm for ventilator-associated events. ${ }^{9}$ This new algorithm was proposed to avoid the discussions on length of ventilation or inaccuracy of microbiological 
detection: new VAP surveillance on the basis of the infection-related ventilator-associated complication definition. ${ }^{9}$ In this issue of Respiratory CARE, Piryapatsom et $\mathrm{al}^{10}$ aimed to evaluate the accuracy and validity of this infection-related ventilator-associated complication algorithm for detecting VAP according to the $2008 \mathrm{CDC} /$ National Healthcare Safety Network (NHSN) definition as the reference diagnosis (VAP-NHSN). ${ }^{8}$ The accuracy of the new infection-related ventilator-associated complication surveillance for detecting VAP-NHSN was determined in highrisk trauma subjects. The sensitivity, specificity, and positive and negative predictive values of infection-related ventilator-associated complication for VAP-NSHN identification were low: $28.12 \%, 91.45,58.06 \%$, and $75.14 \%$, respectively. Subjects with infection-related ventilatorassociated complication, VAP-NHSN, or both had higher morbidity when compared with those without infectionrelated ventilator-associated complication and VAPNHSN. There was no significant difference in clinical outcomes between subjects with VAP-NHSN only and those with both infection-related ventilator-associated complication and VAP-NHSN. The main finding was that the infection-related ventilator-associated complication surveillance algorithm has a low sensitivity and a low positive predictive value for the identification of VAP in highrisk trauma subjects. The algorithm even failed to diagnose about three quarters of subjects with VAP-HNHSN, although it identified subjects requiring increasing ventilator support due to a range of conditions unrelated to VAP. This CDC definition and approach have also performed poorly in other studies, including one in a mixed ICU population not limited to trauma only. ${ }^{11}$ Although a working definition of VAP that includes surveillance could have a theoretical benefit in standardizing supportive intensive care, it fails to do so in real daily life.

Why is the diagnostic accuracy of definitions for many disorders in the ICU and more particularly in ventilated patients such a controversial subject? Just look at sepsis: a complex clinical syndrome involving both infection and a deleterious immune response. A major obstacle to the development of good host-directed therapies in sepsis is the lack of understanding of this host immune response. The problem is exacerbated by poor nonspecific clinical definitions of disease. In a recent study, the need for 2 or more systemic inflammatory response syndrome criteria to define severe sepsis excluded one in 8 otherwise similar subjects with infection, organ failure, and substantial mortality and failed to define a transition point in the risk of death. ${ }^{12}$ Despite being defined as a syndrome over 4 decades ago, recent studies did not validate the most recent Berlin definition of ARDS; neither the stratification by severity nor the $\mathrm{P}_{\mathrm{aO}} / \mathrm{F}_{\mathrm{IO}_{2}}$ at study entry was independently associated with mortality. ${ }^{13}$ Until now, poor definitions have had a profound impact on research in sepsis, ARDS, and VAP, both in epidemiologic studies and in failed clinical trials of host-directed therapies. Therefore, there is an urgent need for better definitions to enable advancement in the field of critical care medicine.

It is remarkable that many of the definitions for disorders and syndromes in critically ill patients lack sensitivity and specificity, although our understanding of the essential nature of these pathological syndromes is continuously increasing. A precise, standardized, and more universal definition of potential disasters, such as sepsis, ARDS, and VAP, would allow comparison of the findings of clinical trials with a greater degree of certainty. A functional definition of VAP would allow early institution of appropriate antibiotics and certain therapeutic modalities that have been proven to have benefits, while omiting useless administration of antibacterial drugs when VAP is not present. It could help to capture a specific patient population for potential recruitment into relevant clinical studies. According to Webster's dictionary, a definition should be universal, proper, clear, and short. ${ }^{14}$ Are we missing the essential clue in VAP that can be used in a universal and clear definition? Until further notice, it might be safer to adhere to an older, far from perfect, but usable definition of VAP: Aren't old friends and old wine best?

Philippe G Jorens MD PhD Department of Critical Care Medicine Antwerp University Hospital University of Antwerp Edegem, Belgium

\section{REFERENCES}

1. Kelly FE, Fong K, Hirsch N, Nolan JP. Intensive care medicine is 60 years old: the history and future of the intensive care unit. Clin Med (Lond) 2014;14(4):376-379.

2. Mietto C, Pinciroli R, Patel N, Berra L. Ventilator associated pneumonia: evolving definitions and preventive strategies. Respir Care 2013;58(6):990-1007.

3. Kalanuria AA, Zai W, Mirski M. Ventilator-associated pneumonia in the ICU. Crit Care 2014;18(2):208.

4. American Thoracic Society, Infectious Diseases Society of America. Guidelines for the management of adults with hospital-acquired, ventilator-associated, and healthcare-associated pneumonia. Am J Respir Crit Care Med 2005;171(4):388-416.

5. Branson RD. The ventilator circuit and ventilator-associated pneumonia. Respir Care 2005;50(6):774-785; discussion 785-787.

6. Luna CM, Aruj P, Niederman MS, Garzón J, Violi D, Prignoni A, et al. Appropriateness and delay to initiate therapy in ventilator-associated pneumonia. Eur Respir J 2006;27(1):158-164.

7. Piskin N, Aydemir H, Oztoprak N, Akduman D, Comert F, Kokturk F, Celebi G. Inadequate treatment of ventilator-associated and hospital-acquired pneumonia: risk factors and impact on outcomes. BMC Infect Dis 2012;12:268-269.

8. Horan TC, Andrus M, Dudeck MA. CDC/NHSN surveillance definition of health care-associated infection and criteria for specific types of infections in the acute care setting. Am J Infect Control. 


\section{Sticking to An Old Definition of VAP}

2008;36(5):309-332. Erratum in: Am J Infect Control. 2008;36(9): 655.

9. Magill SS, Klompas M, Balk R, Burns SM, Deutschman CS, Diekema D, et al. Developing a new, national approach to surveillance for ventilator-associated events. Crit Care Med 2013; 41(11):2467-2475.

10. Piriyapatsom A, Lin H, Pirrone M, De Pascale G, De Lapuerta JC, Bittner EA et al. Evaluation of the infection-related ventilator-associated events algorithm for ventilator-associated pneumonia surveillance in a trauma population. Respir Care 2016;61(3):269-276.

11. Klein Klouwenberg PM, van Mourik MS, Ong DS, Horn J, Schultz $\mathrm{MJ}$, Cremer OL et al. Electronic implementation of a novel surveil- lance paradigm for ventilator-associated events. Feasibility and validation Am J Respir Crit Care Med 2014;189(8):947-955.

12. Kaukonen KM, Bailey M, Pilcher D, Cooper DJ, Bellomo R. Systemic inflammatory response syndrome criteria in defining severe sepsis. N Engl J Med 2015;372(17):1629-1638.

13. Hernu R, Wallet F, Thiollière F, Martin O, Richard JC, Schmitt Z, et al. An attempt to validate the modification of the American-European consensus definition of acute lung injury/acute respiratory distress syndrome by the Berlin definition in a university hospital. Intensive Care Med 2013;39(12):2161-2170.

14. Webster's Dictionary 1913. http://www.webster-dictionary.org/ definition/definition. Accessed January 22, 2016. 